\title{
The Main Anatomical Variations of the Pancreatic Duct System: Review of the Literature and Its Importance in Surgical Practice
}

\author{
Ioannis Dimitriou $^{\mathrm{a}, \mathrm{c}, \mathrm{d}}$, Anastasios Katsourakis $^{\mathrm{b}}$, Eirini Nikolaidou $^{\mathrm{b}}$, George Noussios $^{\mathrm{c}}$
}

\begin{abstract}
Anatomical variations or anomalies of the pancreatic ducts are important in the planning and performance of endoscopic retrograde cholangiopancreatography (ERCP) and surgical procedures of the pancreas. Normal pancreatic duct anatomy occurs in approximately $94.3 \%$ of cases, and multiple variations have been described for the remaining $5.7 \%$. The purpose of this study was to review the literature on the pancreatic duct anatomy and to underline its importance in daily invasive endoscopic and surgical practice. Two main databases were searched for suitable articles published from 2000 to 2017, and results concerning more than 8,200 patients were included in the review. The most common anatomical variation was that of pancreas divisum, which appeared in approximately $4.5 \%$ of cases.
\end{abstract}

Keywords: Pancreatic duct; Anatomy; Anatomic; Variations; Anomalies

\section{Introduction}

Anatomical variations, or anomalies, of the pancreas and pancreatic duct system are not unusual, which are usually asymptomatic, and may not be discovered until adulthood [1-3]. Sometimes, general signs and symptoms such as abdominal pain, nausea, and vomiting may be due to recurrent pancreatitis or gastric outlet obstruction caused by a congenital variant of the pancreas or the pancreatic duct system $[1,4]$. These diagnoses should be considered and excluded after proper imaging examinations. Normal pancreatic duct variants and variations

Manuscript submitted January 6, 2018, accepted February 15, 2018

aDepartment of Anatomy in the Department of Physical Education and Sports Science (Serres), Aristotle University of Thessaloniki, Thessaloniki, Greece bepartment of Surgery, Agios Dimitrios General Hospital, Thessaloniki, Greece 'Laboratory of Anatomy in the Department of Physical Education and Sports Science (Serres), Aristotele University of Thessaloniki, Thessaloniki, Greece ${ }^{\mathrm{d} C}$ Corresponding Author: Ioannis Dimitriou, Laboratory of Anatomy in the Department of Physical Education and Sports Science (Serres), Aristotele University of Thessaloniki, Kanari 7 Stavroupoli, Thessaloniki 56430, Greece. Email: johndim577@yahoo.com of pancreas divisum are frequently discovered as incidental findings on clinical imaging [5]. The most common imaging modalities that evaluate the pancreas and the pancreatic duct system are the computed tomography (CT) scan of the abdomen, the magnetic resonance cholangiopancreatography (MRCP), and the endoscopic retrograde cholangiopancreatography (ERCP). CT of the abdomen is very useful in finding and imaging pathologies of the pancreas, such as acute or chronic pancreatitis and pancreatic tumors, but it is not very good at imaging the pancreatic duct system. MRCP is a very accurate, noninvasive technique for visualizing the pancreatic and biliary duct system without the side effects of a contrast agent and without the risk of causing acute pancreatitis [2, 4-6]. ERCP is considered the best diagnostic tool for imaging the pancreatic and biliary duct system because it produces better results in imaging; however, the radiation it uses, is invasive, and carries the risk of causing acute pancreatitis in a small group of patients $[4,7]$.

Anatomic variations and anomalies of the pancreatic duct system that can be diagnosed with these techniques include configuration variations (bifid configuration with dominant main pancreatic duct (MPD), dominant accessory pancreatic duct (APD) without divisum, pancreas divisum, absent APD, ansa pancreatica, and cystic dilations of terminal portions of the MPD and APD), course variations (descending, loopshaped or ring-shaped, vertical, and sigmoid), duplication anomalies, and anomalous pancreaticobiliary ductal junction (APBU) $[1,2,8]$.

Likewise, these techniques can diagnose anomalies of the pancreas such as annular pancreas, ectopic pancreas, agenesis and hypoplasia of the pancreas, and accessory pancreatic lobe $[1,2,8]$. The frequency of the pancreatic duct variations in a general population and the clinical relevance has not been well investigated $[2,5]$. Diagnosing these variants is important because they may be a cause of pancreatitis or gastric outlet obstruction that can be corrected operatively $[1,2,4]$. This knowledge is also important when ERCP is planned for diagnostic or therapeutic reasons because it may help in treatment planning and prevent injuries of the pancreas and the pancreatic ducts during surgery or ERCP procedures [2, 4].

\section{Literature Search}

A systematic search of the medical scientific literature was car- 

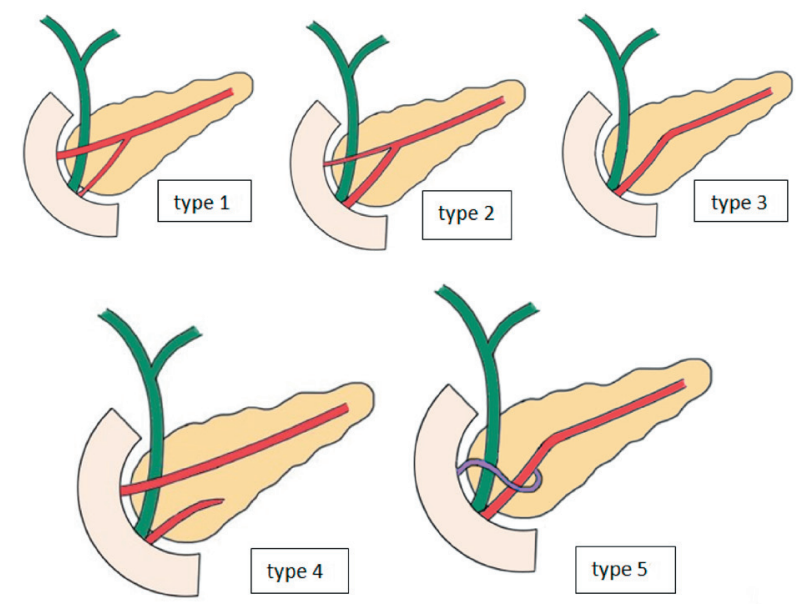

Figure 1. Drawings showing different types of pancreatic duct configuration.

ried out using PubMed and Google Scholar databases for 2000 - 2017 in order to access all the published articles involving the anatomic variations of the pancreatic duct system.

The following terms and their combinations were used: "pancreatic duct," "anatomy," "anatomic," "variations," and "anomalies." All the articles considered relevant were included in the research, and all their relevant references were checked to find any missing studies. The following inclusion criteria were used for this study: 1) the article was original, a review, or a prospective study, 2) the article was written in English.
The following exclusion criteria were used for this study: 1) articles written in a language other than English, 2) articles concerning populations overlapping one another, 3) articles concerning animal subjects, not human subjects, 4) articles that reported case reports and small case series.

The pancreatic duct system classification was defined using the conventional pancreatic ductal anatomy (Fig. 1). We investigated the type of study (systematic review, retrospective, or prospective study), the year of publication, and the kind of subjects (healthy people, patients, or cadavers) in every study considered eligible for our research.

\section{Literature Retrieved}

Ten articles were retrieved using the described search strategy. All articles were analyzed in the review (Fig. 2).

A total of 8,260 patients were analyzed in our study, of which $94.3 \%$ of cases displayed normal anatomy (types 1 3 ), $4.5 \%$ displayed pancreas divisum, and $1.2 \%$ were reported with rare anomalies. The studies that were considered eligible in our review consisted of five retrospective studies, four prospective studies, and one multicenter retrospective study (Table $1,[3-5,7,9-14])$.

\section{Discussion}

The development of the human pancreas embryology is complex, resulting in many different congenital pancreaticobiliary

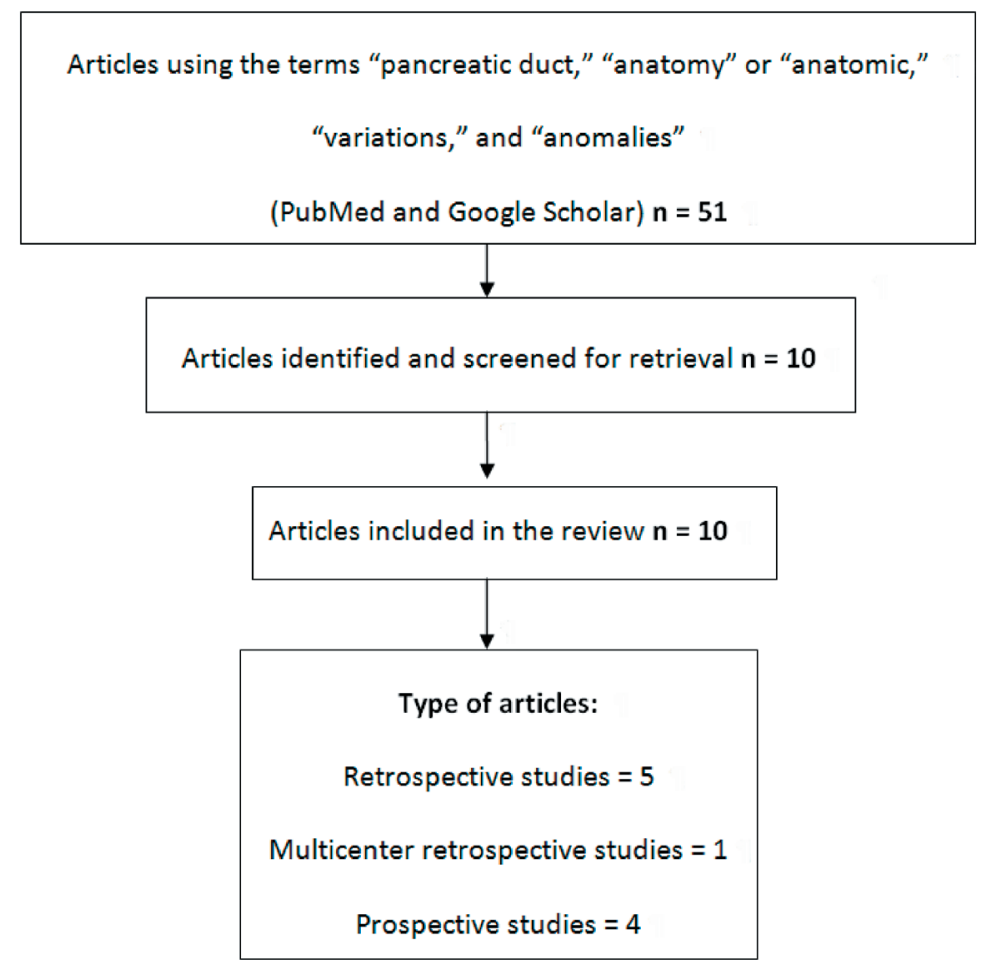

Figure 2. Summary of the literature research. 
Table 1. Classification of the Articles According to the Number and Type of Patients, Type of Investigation, and Type of Study [3-5, $7,9-14]$

\begin{tabular}{lllll}
\hline Author & No. of subjects & Type of subjects & Type of investigation & Type of article \\
\hline Filippo et al [3] & 350 & Living patient & MRCP & Prospective study \\
Adibelli et al [4] & 1,158 & Living patient & MRCP & Retrospective cohort study \\
Bulow et al [5] & 927 & Healthy volunteers & Stimulating MRCP & Prospective study \\
Bang et al [7] & 582 & Living patient & ERCP & Retrospective cohort study \\
Kamisawa et al [9] & $256 / 3,210^{*}$ & Living patient & ERCP & Retrospective cohort study \\
Shahriah et al [10] & 65 & Cadaver & Autopsy of specimens & Prospective study \\
Kim HJ et al [11] & $4,097 / 10,243^{*}$ & Living patient & ERCP & Multicenter retrospective cohort study \\
Oracz et al [12] & 300 & Living patient & Medical records (non specified) & Retrospective cohort study \\
Uomo et al [13] & $485 / 650^{*}$ & Living patient & ERCP & Retrospective cohort study \\
Prasanna et al [14] & 40 & Cadaver & Autopsy of specimens & Prospective study \\
\hline
\end{tabular}

${ }^{*}$ Only some of the patients included in the article were suitable for imaging of the pancreatic duct system. ERCP: endoscopic retrograde cholangiopancreatography; MRCP: magnetic resonance cholangiopancreatography.

variations $[1,3]$. The human pancreas develops from the ventral and dorsal pancreatic buds whose fusion causes the anastomosis of the pancreatic ducts at the 6th to 7 th embryological week $[1,8,9,15,16]$. The ventral pancreatic duct and the distal part of the dorsal pancreatic duct are anastomosed and form the MPD of Wirsung, while the proximal part of the dorsal pancreatic duct becomes the APD of Santorini $[1,9,15]$. The major pancreatic duct opens into the ampulla of Vater, and the minor pancreatic duct opens into the minor papilla $[1,9$, 10]. Any alteration of the described pancreatic duct anatomy is considered a variation, or anomaly, of the pancreatic duct system.

In our study, we used the conventional classification of the pancreatic duct system, which classifies the pancreatic duct anatomy into five types. Type 1 is characterized by a bifid configuration with Wirsung as the dominant duct, type 2 is characterized by a bifid configuration with Santorini as the dominant duct, type 3 is characterized by a rudimentary nondraining or absent duct of Santorini, type 4 is characterized as pancreas divisum, and type 5 is characterized as ansa pancreatica (Fig. 1) $[1,2,4]$.

In many of the articles that we found in the literature, the authors did not use this kind of classification; instead, they used the term "normal pancreatic duct" to describe a pancreas that could be classified as type 1,2 , or 3 . Therefore, we could not separate the patients between these three groups, and we presented them as a unique type of normal pancreatic duct anatomy (Table 2 and 3, [3-5, 7, 9-14]). In addition, some authors used a different type of pancreatic duct classification (e.g., Bang et al (2006) [7]; Bulow et al (2014) [5]), and the correspondence was made according to the descriptions they used for the type of pancreatic duct they studied.

Our study comprises a review of PubMed and Google Scholar databases. It looks into the anatomical variations of

Table 2. Classification of the Articles According to the Number of Patients, Normal Pancreatic Duct Anatomy, and Variations [3-5, $7,9-14]$

\begin{tabular}{llllll}
\hline Author & No. of subjects examined & Type of subjects & No. of normal & Pancreas divisum & Other specific types of anomalies \\
\hline Filippo et al [3] & 350 & Living patient & 321 & 18 & 11 \\
\hline Adibelli et al [4] & 1,158 & Living patient & 1,091 & 54 & 13 \\
Bulow et al [5] & 927 & Healthy volunteers & 838 & 89 & - \\
Bang et al [7] & 582 & Living patient & 531 & 19 & 32 \\
Kamisawa et al[9] & $256 / 3,210$ & Living patient & 175 & 81 & - \\
Shahriah et al [10] & 65 & Cadaver & 55 & 9 & 1 \\
Kim HJ et al [11] & $4,097 / 10,243$ & Living patient & 4,054 & 40 & 3 \\
Oracz et al [12] & 300 & Living patient & 252 & 33 & 22 \\
Uomo et al [13] & $485 / 650$ & Living patient & 437 & 26 & - \\
Prasanna et al [14] & 40 & Cadaver & 38 & 2 & $97(1.2 \%)$ \\
Total & 8,260 & 8 living patients & $7,792(94.3 \%)$ & $371(4.5 \%)$ & \\
& & 1 healthy volunteers & & \\
\hline
\end{tabular}


Table 3. Classification of Patients According to the Type of the Pancreatic Duct Course and Configuration [3-5, 7, 9-14]

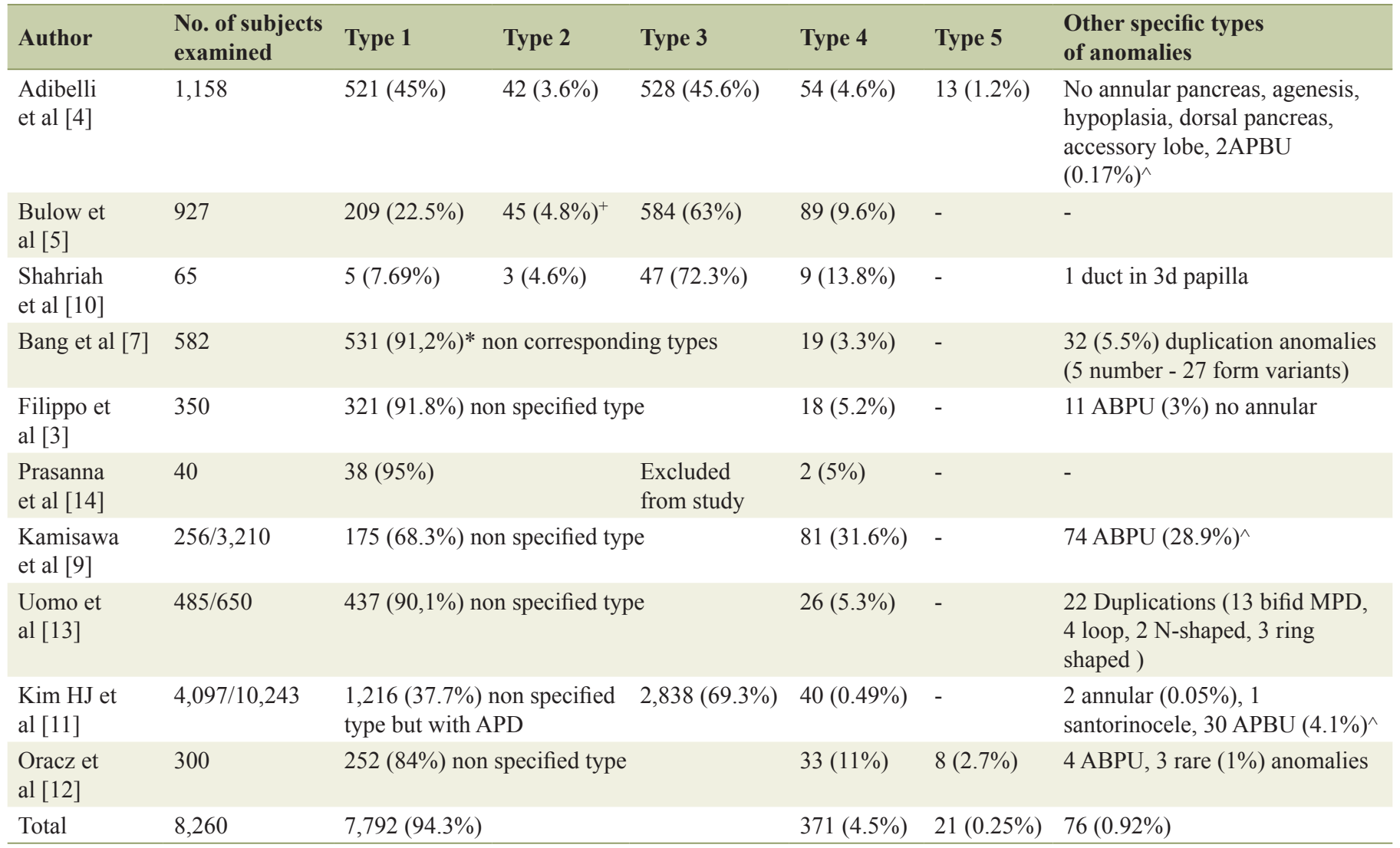

${ }^{*}$ Patients with type 1, type 2 and type 3 were not discriminated due to the different type of classification. ${ }^{+}$Patients that could not be classified in one of the described types according to the classification that was used in this article. ^Patients with APBU were not discriminated from the types 1 to 5 but they were reported separately.

the pancreatic duct system as they are described in 10 eligible articles. A total of 8,260 human patients (adults and children) were included. We used the classification of the pancreatic duct system that was described above as the most complete and representative. Normal pancreatic duct anatomy as it is defined by types 1, 2, and 3 was described in 7,792 patients $(94.3 \%)$ (Tables 2 and 3, [3-5, 7, 9-14]) (Fig. 3).

The most common variant was pancreas divisum (type 4), which was described in 371 patients $(4.5 \%)$. Pancreas divisum results from failure of fusion or abnormal fusion of the dorsal and ventral ducts of the pancreas during embryological development $[1,2,9]$. It occurs in approximately $3 \%$ to $14 \%$ of the population worldwide $[2,4,5,8,17,18]$. However, low percentages, less than $1 \%$, have been reported in studies from Asia [11, 19], whereas in Europe and the United States, its prevalence is between $2 \%$ and $10 \%[4,5,11,16,20,21]$. Pancreas divisum has three subtypes: type 1( no communication between dorsal and ventral pancreatic duct), type 2 (absent ventral duct), and type 3 (small communication between the two branches) $[1,4,11,17]$. Only four articles separated patients according to the type of pancreas divisum they had (Table 4, [4, 5, 7, 9]) (Fig. 4).

In our study, other anomalies of the pancreatic duct system were described in 97 patients $(1.2 \%)$ and included ansa pancre- atica $(0.25 \%)$, annular pancreas, duplication anomalies, santorinicele, APBU, and unspecified rare anomalies. Only three articles reported 36 patients having ansa pancreatica $(0.4 \%)$ [ $7,12]$, and only one article reported two cases of annular pancreas and a single patient with santorinicele [11]. Duplication anomalies were reported in only two articles. The first one, by Bang et al (2006), described 32 patients with duplication anomalies who were not separately described from types 1 - 5 $[7,13]$. The second article, by Uomo et al (1995), described 22 patients who were separated from types 1 - 5 as rare anomalies [13]. APBU was described as a variant in five studies that reported 121 patients $(1.4 \%)$ as having this anomaly $[3,4,9$, $11,12]$. Only one article reported an APD that opened into the third papilla [10]. Two articles studied cadavers and reported results from human pancreases that included descriptions of the pancreatic ducts that were different from the usual pancreatic model used by clinical doctors and also used in this review $[10,14]$. To include these two studies in our review, we converted their results to the conventional types (1 - 5) using their descriptions of the pancreatic ducts.

Anatomical anomalies are considered a predisposition factor to illnesses of the pancreas such as pancreatitis or impairment of the pancreatic exocrine function [6, 22]. Pancreas divisum is especially considered by many authors as a risk 


\section{Pancreatic duct type}

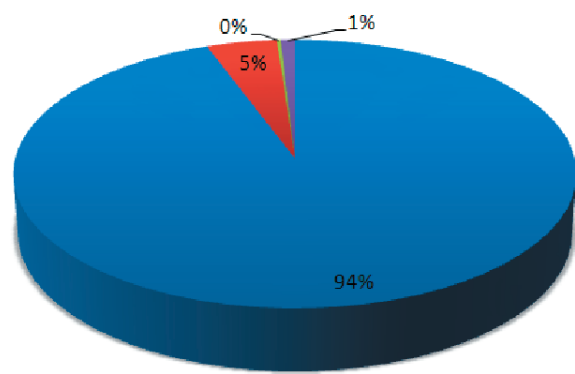

Figure 3. Schematic presentation of the presence of the pancreatic duct types.

factor for chronic pancreatitis $[6,22]$. In this review, we discovered that, in most cases, there was no correlation between pancreas divisum and pancreatic disease $[4,5,8,15,21]$, even though some authors still support that pancreas divisum might play a role in chronic pancreatitis $[1,17,20,23]$. In addition, Oracz et al (2006) demonstrated that children suffering from chronic pancreatitis are found to have pancreas divisum more frequently and have the worst outcomes [12]. However, many authors support that subjects with pancreas divisum are usually asymptomatic, and the percentages of chronic pancreatitis do not differ between these patients and those with normal pancreatic duct anatomy $[2,5,8]$. On the contrary, annular or circumportal pancreas is considered a strong positive factor for postoperative pancreatic fistula after a pancreatectomy and should be taken into serious consideration before pancreatic surgery $[2,4,16,24]$. Ansa pancreatica is also considered a relevant factor to the onset of chronic pancreatitis [4]. Morphology of the MPD plays a significant role in pancreatic anastomoses, and an investigation to determine its correlations is necessary before pancreatic procedures [25].

Our study has several limitations. First, this was a systematic review of collected data from several articles that used different classifications for the description of the pancreatic duct system in the patients they studied. Second, the studies were either prospective or retrospective, and the subjects they studied were from different populations. For example, two articles used human cadavers, one examined healthy people, and the remaining seven studies involved patients who suffered from a disease of the pancreas or the bile duct system (e.g., choledocholithiasis) (Table 1). Finally, some authors made discriminations between different rare anomalies, while others included them as types 1 - 5 together with subjects that did not have

Table 4. Evaluation of Pancreas Divisum and Its Types

\begin{tabular}{llll}
\hline Pancreas divisum & Type 1 & Type 2 & Type 3 \\
\hline Adibelli et al [4] & 24 & 20 & 10 \\
Bang et al [7] & 12 & - & 7 \\
Bulow et al [5] & 67 & - & 89 \\
Kamisawa et al [9] & 16 & 10 & 15 \\
Total & $119(44 \%)$ & $30(11.11 \%)$ & $121(44.8 \%)$ \\
\hline
\end{tabular}

\section{Pancreas Divisum}

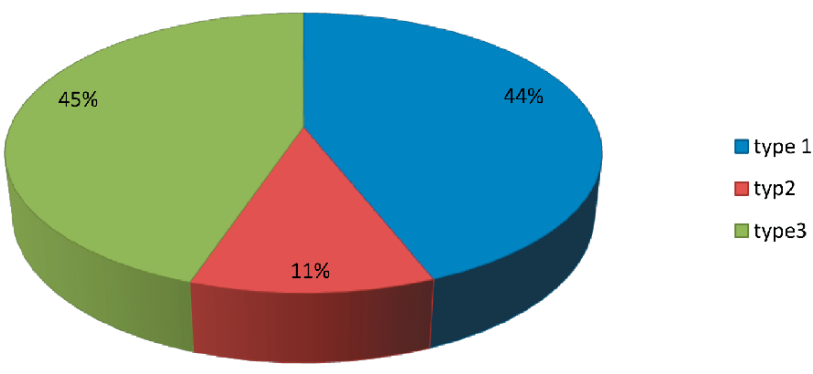

Figure 4. Schematic presentation of the percentages of pancreas divisum types.

that type of variation (e.g., APBU patients sometimes were referred as a separate group, and other times their presence was overlapped by other anatomical types). To overcome this possible bias, a prospective multicenter registration strategy using a large number of subjects is necessary.

\section{Conclusions}

Knowledge of the anatomical variations of the pancreatic ducts is of great importance in general surgery, especially in pancreatic surgery, and is essential to help surgeons perform pancreatic anastomoses safely and effectively $[8,11]$. This knowledge is also crucial with regard to invasive gastroenterology and the practicing of ERCP as a diagnostic and therapeutic procedure $[11,14,26]$. In conclusion, surgeons, gastroenterologists, and radiologists should be aware of these situations in order to avoid iatrogenic damage of the patients [3]. Rare anatomical variations of the pancreatic ducts, including pancreas divisum, occur less than $6 \%$ of the time, but a large prospective cohort study is necessary to determine the real incidence of the relevant anatomical anomalies and their clinical importance.

\section{Acknowledgments}

We would like to thank Athanasiadis Efthimios for his kind offer of the drawings that were used in this article.

\section{Conflict of Interest}

The authors declare that they do not have any conflict of interest.

\section{References}

1. Arora A, Rajesh S, Mukund A, Patidar Y, Thapar S, Arora A, Bhatia V. Clinicoradiological appraisal of "paraduodenal pancreatitis': Pancreatitis outside the pancreas! Indian J Radiol Imaging. 2015;25(3):303-314. 
2. Turkvatan A, Erden A, Turkoglu MA, Yener O. Congenital variants and anomalies of the pancreas and pancreatic duct: imaging by magnetic resonance cholangiopancreaticography and multidetector computed tomography. Korean J Radiol. 2013;14(6):905-913.

3. De Filippo M, Calabrese M, Quinto S, Rastelli A, Bertellini A, Martora R, Sverzellati N, et al. Congenital anomalies and variations of the bile and pancreatic ducts: magnetic resonance cholangiopancreatography findings, epidemiology and clinical significance. Radiol Med. 2008;113(6):841-859.

4. Adibelli ZH, Adatepe M, Imamoglu C, Esen OS, Erkan $\mathrm{N}$, Yildirim M. Anatomic variations of the pancreatic duct and their relevance with the Cambridge classification system: MRCP findings of 1158 consecutive patients. Radiol Oncol. 2016;50(4):370-377.

5. Bulow R, Simon P, Thiel R, Thamm P, Messner P, Lerch MM, Mayerle J, et al. Anatomic variants of the pancreatic duct and their clinical relevance: an MR-guided study in the general population. Eur Radiol. 2014;24(12):31423149.

6. Vitellas KM, Keogan MT, Spritzer CE, Nelson RC. MR cholangiopancreatography of bile and pancreatic duct abnormalities with emphasis on the single-shot fast spinecho technique. Radiographics. 2000;20(4):939-957; quiz 1107-1108, 1112.

7. Bang S, Suh JH, Park BK, Park SW, Song SY, Chung JB. The relationship of anatomic variation of pancreatic ductal system and pancreaticobiliary diseases. Yonsei Med J. 2006;47(2):243-248.

8. Brar NS, Bajwa RS. Prospective observational study on pancreatic duct system diversity in different pancreatic diseases. International Surgery Journal. 2017;4(10):33303337.

9. Kamisawa T, Okamoto A. Pancreatographic investigation of pancreatic duct system and pancreaticobiliary malformation. J Anat. 2008;212(2):125-134.

10. Shahriah S, Nurunnabi ASM, Johora F, Siddiqua D, Ara S. Cadaveric study of the anatomical variations of the accessory pancreatic duct. Journal of Bangladesh Society of Physiologist. 2015;9(2):83-88.

11. Kim HJ, Kim MH, Lee SK, Seo DW, Kim YT, Lee DK, Song SY, et al. Normal structure, variations, and anomalies of the pancreaticobiliary ducts of Koreans: a nationwide cooperative prospective study. Gastrointest Endosc. 2002;55(7):889-896.

12. Oracz G, Oralewska B, Pertkiewics J, Teisseyre M, Ryzko J, Socha J. Chronic pancreatitis associated with anatomic anomalies of pancreatic duct in children. Journal of Pediatric Gastroenterology and Nutrition. 2006;42(5):E58.
13. Uomo G, Manes G, D’Anna L, Laccetti M, Di Gaeta S, Rabitti PG. Fusion and duplication variants of pancreatic duct system. Clinical and pancreatographic evaluation. Int J Pancreatol. 1995;17(1):23-28.

14. Prasanna LC, Rajagopal KV, Thomas HR, Bhat KM. Accessory pancreatic duct patterns and their clinical implications. J Clin Diagn Res. 2015;9(3):AC05-07.

15. Mortele KJ, Rocha TC, Streeter JL, Taylor AJ. Multimodality imaging of pancreatic and biliary congenital anomalies. Radiographics. 2006;26(3):715-731.

16. Mitchell CJ, Lintott DJ, Ruddell WS, Losowsky MS, Axon AT. Clinical relevance of an unfused pancreatic duct system. Gut. 1979;20(12):1066-1071.

17. Yu J, Turner MA, Fulcher AS, Halvorsen RA. Congenital anomalies and normal variants of the pancreaticobiliary tract and the pancreas in adults: part 2, Pancreatic duct and pancreas. AJR Am J Roentgenol. 2006;187(6):15441553 .

18. Leyendecker JR, Elsayes KM, Gratz BI, Brown JJ. MR cholangiopancreatography: spectrum of pancreatic duct abnormalities. AJR Am J Roentgenol. 2002;179(6):14651471.

19. Ueno E, Takada Y, Yoshida I, Toda J, Sugiura T, Toki F. Pancreatic diseases: evaluation with MR cholangiopancreatography. Pancreas. 1998;16(3):418-426.

20. Chalazonitis NA, Lachanis BS, Laspas F, Ptohis N, Tsimitselis G, Tzovara J. Pancreas divisum: magnetic resonance cholangiopancreatography findings. Singapore Med J. 2008;49(11):951-954; quiz 955.

21. Bertin C, Pelletier AL, Vullierme MP, Bienvenu T, Rebours V, Hentic O, Maire F, et al. Pancreas divisum is not a cause of pancreatitis by itself but acts as a partner of genetic mutations. Am J Gastroenterol. 2012;107(2):311317.

22. Puente SG, Bannura GC. Radiologiacal anatomy of the biliary tract: variations and congenital abnormalities. World J Surg. 1983;7:271-276.

23. Bernard JP, Sahel J, Giovannini M, Sarles H. Pancreas divisum is a probable cause of acute pancreatitis: a report of 137 cases. Pancreas. 1990;5(3):248-254.

24. Ohtsuka T, Mori Y, Ishigami K, Fujimoto T, Miyasaka Y, Nakata K, Ohuchida K, et al. Clinical significance of circumportal pancreas, a rare congenital anomaly, in pancreatectomy. Am J Surg. 2017;214(2):267-272.

25. Shukla PJ, Sakpal SV, Maharaj R. Does pancreatic ductal anatomy play a role in determining outcomes of pancreatic anastomoses? Med Hypotheses. 2011;76(2):230-233.

26. Kamisawa T, Honda G, Kurata M, Tokura M, Tsuruta K. Pancreatobiliary disorders associated with pancreaticobiliary maljunction. Dig Surg. 2010;27(2):100-104. 